\title{
A Short Term Outcome of Laparoscopic Hand Sewn and Stapled Gastrojejunostomy in Gastric Outlet Obstruction - An Observational Study
}

\author{
Mushtaq C*, Mumtaz DW, Peer HAM, Awhad MY, Wasim R, Syed SA, \\ Zubair GL and Aabid RB \\ Department of surgery, Government Medical College Srinagar, India
}

*Corresponding author: Mushtaq Chalkoo Associate Professor, Laparo-endoscopic surgeon, Government Medical College Srinagar, Email: mushtaq_chalkoo@rediffmail.com

\author{
Research Article \\ Volume 2 Issue 2 \\ Received Date: April 09, 2018 \\ Published Date: April 28, 2018
}

\section{Abstract}

Background: Gastric Outlet Obstruction is a disease condition, where there is inability of gastric contents to go beyond the proximal duodenum. The major cause is known to be malignancy, especially in the developing world. Palliative treatment is important and required for patients with unrestable primary gastric outlet tumours or even with metastatic lesions. We took up laparoscopic gastrojejunostomy as a study to observe the results of laparoscopic hand sewn and laparoscopic stapled anastomosis with reference to operative time, anastomosis time, return of bowel sounds, post operative complications and total hospital stay.

Methods: This study was prospective, observational, hospital based conducted in the department of General and Minimal Access Surgery, Government Medical College Srinagar between June 2014 to September 2016.40 patients were selected and grouped into 20 each as A \& B. Laparoscopic hand sewn anastomosis was performed in Group A and laparoscopic stapled anastomosis was performed in Group B and different parameters were observed in each group.

Results: The results of our study were conclusive. The mean operative time in minutes in laparoscopic hand sewn anastomosis (Group A) was 101.2 with S.D of 8.94 and in laparoscopic stapled anastomosis (Group B) was 90.8 with S.D of 7.97. The time of anastomosis in minutes in laparoscopic hand sewn anastomosis (Group A) was 22.3 with S.D of 1.86 and in laparoscopic stapled anastomosis (Group B) was 17.4 with S.D of 2.48. The mean hospital stay in days in laparoscopic hand sewn anastomosis (Group A) was 8.4 with S.D of 0.89 and in laparoscopic stapled anastomosis (Group B) was 7.9 with S.D of 0.91. The appearance of bowel sound in days in laparoscopic hand sewn anastomosis (Group A) was 2.6 with S.D of 0.59 and in laparoscopic stapled anastomosis (Group B) was 2.3 with S.D of 0.47. In laparoscopic hand sewn anastomosis (Group A) the complication rate was 15\% while as in laparoscopic stapled anastomosis (Group B) was complication rate was $10 \%$. 


\section{International Journal of Surgery \& Surgical Techniques}

Conclusion: Stapling devices in surgery are a versatile tool in the armamentarium of a surgeon. Anastomosis in laparoscopic stapled group took less time and thus decreased total operative time.

Keywords: Laparoscopy; Gastrojejunostomy; Techniques

Abbreviations: GOO: Gastric Outlet Obstruction; PUD: Peptic Ulcer Disease; QOL: Quality of Life.

\section{Introduction}

Gastric outlet obstruction (GOO) is a term used to describe a condition characterized by the inability of gastric contents to go beyond the proximal duodenum. Gastric outlet obstruction (GOO) is a misnomer expression as numerous cases include duodenal or extra luminal disease, rather than an isolated gastric disease. The obstruction may be partial or complete [1-3]. Apart from the mechanical impediment of the flow of gastric contents, there are other concerns in patients with GOO, such as malnutrition and electrolyte imbalance [4]. The incidence of GOO is not precisely known. Until the discovery of proton pump inhibitors and $\mathrm{H} 2$ blockers, the major cause of GOO was peptic ulcer disease (PUD). The discovery of Helicobacter pylori and developing an effective treatment for the same has resulted in fewer cases of PUD presenting with GOO $(<5 \%)$; however, in the modern era, the major cause is known to be malignancy, especially in the developed world [5].

The causes of gastric outlet obstruction can be categorized as gastric and extra gastric in origin. Chronic peptic ulceration, gastric adenocarcinoma, GIST, gastric lymphoma, tuberculosis and amyloidosis comprise gastric causes. While as periampullary carcinoma, hepato-biliary cancer, pancreatic cancer, pancreatic pseudo cyst, colon cancer and neuroendocrine tumors are extra-gastric causes that can present with gastric outlet obstruction.

Malignant GOO is a common condition among locally advanced gastric cancer patients and can lead to significant morbidity, including nausea, vomiting, abdominal pain, dehydration, malnutrition, and weight loss. Not surprisingly, these clinical symptoms have a negative impact on quality of life (QOL) [6]. To avoid the disastrous consequences of malignant GOO, appropriate treatment is indispensable, which enables not only an amelioration of the patient's QOL but also the commencement of chemotherapy [7]. These treatments are included in the first-line regimen for unrespectable gastric cancer recommended in the Japanese gastric cancer treatment guidelines [8].

The most common surgical procedures performed for GOO related to PUD are vagotomy and antrectomy, vagotomy and pyloroplasty, truncalvagotomy and Gastrojejunostomy, pyloroplasty, and laparoscopic variants of the afore mentioned procedures [9].

The role of the laparoscopic approach in the treatment of GOO is under investigation and may represent a valid form of therapy with low morbidity. The experience of several international centers has been published. One group in China performed laparoscopic truncalvagotomy and Gastrojejunostomy for GOO related to PUD, with nearly complete resolution of symptomatology. The investigators reported no conversions to open procedure or mortalities. Twenty-seven percent of patients did experience transiently delayed gastric emptying, which resolved with conservative measures [10].

The gastric outlet obstruction bypass (Gastrojejunostomy) can be performed by both open and laparoscopic technique. Gastrojejunostomy is a surgical procedure in which an anastomosis is created between the stomach and the proximal loop of the jejunum. This is usually done either for the purpose of draining the contents of the stomach or to provide a bypass for the gastric contents. Gastrojejunostomy can be done via either an open or a laparoscopic approach.

The ante colic position may be preferred because it is technically easy. However, when Gastrojejunostomy is performed for malignant disease, whether after gastrectomy or in a palliative setting, the ante colic position is preferred to the retro colic position. When the retro colic position is used, the anastomosis is at risk of obstruction owing to enlargement of the lymph nodes or serosal implants in the transverse mesocolon. In a retrocolic gastro jejunostomy, there is potential to form a gastrojejuno-colic fistula.

At present, laparoscopic gastrojejunostomy is preferred to open gastrojejunostomy whenever possible 


\section{International Journal of Surgery \& Surgical Techniques}

and feasible, especially in a palliative setting for advanced malignancy.

\section{Aims and Objectives}

Patients were divided into two groups and surgical outcome of laparoscopic hand sewn anastomosis and stapled anastomosis in gastric outlet obstruction was evaluated.

The present study was prospective in nature and aimed to observe the following parameters in either group.

a) Operative time of procedure

b) Time for making anastomosis.

c) Total hospital stay.

d) Return of bowel sound.

e) Post-operative complications.

\section{Materials and Methods}

This study was prospective, observational, hospital based study conducted in the department of general surgery government medical college, Srinagar between June 2014 to September 2016. It was undertaken after getting a clearance by our college ethical committee board. All the cases were performed by a single surgeon. Forty patients were selected randomly, who were admitted for elective surgery for gastric outlet obstruction and after proper evaluation, build up and after anesthetic clearance these patients were posted for diagnostic laparoscopy and subsequent laparoscopic Gastrojejunostomy. Forty patients of gastric outlet obstruction were randomly divided into two groups. These were labeled as group A and group B without giving any choice to the patient. Group A consisted of 20 patients and group B consisted of 20 patients. The Patients in group A were subjected to laparoscopic Gastrojejunostomy with hand sewn anastomosis (Extracorporeal suture anastomosis). Those in group B were subjected to laparoscopic gastrojejunostomy with stapled anastomosis. If intraoperatively, the tumor was found to be respectable, then bilroth II operation was done laparoscopically in both groups and anastomosis done according to the group to which the patient was assigned. However those patients where resection was done were not included in the study.

\section{Inclusion Criteria}

The patient characteristics that were included in the study were as
1. Age group $>20$ years as gastric malignancy is uncommon before this age group.

2. Both sexes.

3. Patients with gastric outlet obstructions due to malignant conditions of stomach.

4. Patients with gastric outlet obstruction due to any other benign conditions of stomach.

\section{Exclusion Criteria}

Following patients were excluded from this study

a) All those Patients who did not give consent for surgery after explaining the consequence and outcome of procedure.

b) Patients in whom after doing diagnostic laparoscopy, the growth was resectable and needed open laparotomy for definitive surgery without any regard to co morbidities.

\section{Pre-0perative Preparation}

a) Patients were evaluated for malignant gastric outlet obstruction on OPD basis and all baseline investigations were done.

b) The patients were thoroughly optimized while being admitted in the ward taking special care to their build up especially haemoglobin, plasma proteins, electrolyte corrections and replenishing their clotting status.

c) We followed a strict protocol of nil oral scheme for minimum 2 days before surgery by putting a Ryles tube and preparing stomach by conventional stomach washes.

d) Pre-anesthetic medication was given on the night before surgery.

e) Part preparation was done in the morning, on the day of surgery.

f) Informed and written consent was taken prior to the performance of each procedure.

g) (Ceftriaxone 1gm) was given to each patient.

\section{Operative Technique}

With the patient under general anesthesia, we performed laparoscopic gastrojejunostomy. Laparoscopic Hand sewn anastomosis was done in patients belonging to group A and stapled anastomosis in patients belonging to group B. Patients were placed in supine position. The required instruments and port positions are shown in 


\section{International Journal of Surgery \& Surgical Techniques}

Figures 1 and 2. Three ports were used and all ports were in mid clavicular line. Upper port was $12 \mathrm{mmport}$, situated $2 \mathrm{~cm}$ from right costal margin in mid clavicular line. This is the port for introduction of staplers, but in case of laparoscopic hand sewn (sutured) anastomosis, $12 \mathrm{~mm}$ was replaced with $5 \mathrm{~mm}$ port. Second port was 10 $\mathrm{mm}$ port (optical port) that lies in midclavicular line $5 \mathrm{~cm}$ just below the $12 \mathrm{~mm}$ port. Third port was $5 \mathrm{~mm}$ and lie $5 \mathrm{cmbelow} 10 \mathrm{~mm}$ port in mid clavicular line.

After introduction of ports, the diagnostic laparoscopy was performed and MNT staging was done. The liver, pelvis and surrounding structures were examined for metastasis. As cites was recorded and examined. Finally, the tumor size with extent was evaluated and local advancement confirmed. After MNT staging, if conditions favored resectability, the patient was subjected to resection and definitive surgery was performed. If the staging favored unresettable growth, laparoscopic gastrojejunostomy was done. First duodenojejunal flexure was identified and 15 to $20 \mathrm{~cm}$ distal to DJ (duodenojejunal flexure) jejunal loop was mobilized along the gastric curvature. Then two stay sutures, approximating jejunum with stomach was taken 6cmapart from each other. Then jejunostomy and gastronomy was created by energy source. Then in case of stapled anastomosis two ends of stapler were kept in jejunostomy and gastronomy openings and fired and in case of hand sewn anastomosis vicryl / V-Loc (polydiaxonone) was used to fashion anastomosis by laparoscopic hand sewn technique (collage A, B).

Several factors in postoperative outcomes (operative time, the time for anastomosis, postoperative hospital stay, first flatus time, complications, symptoms related to anastomosis) were evaluated and observed between the two groups. Gastric cancer stage was classified according to the $7^{\text {th }}$ edition of American joint committee on cancer staging criteria [11]. All the values were expressed as mean \pm standard deviations.

\section{Observation and Results}

This prospective study was carried out in department of general surgery at government medical college, Srinagar, J\&K, India. A total of 40 patients were included in the study and distributed into two groups (Group A: Laparoscopic hand sewn anastomosis, Group B: Laparoscopic stapled anastomosis) and following observations were made.

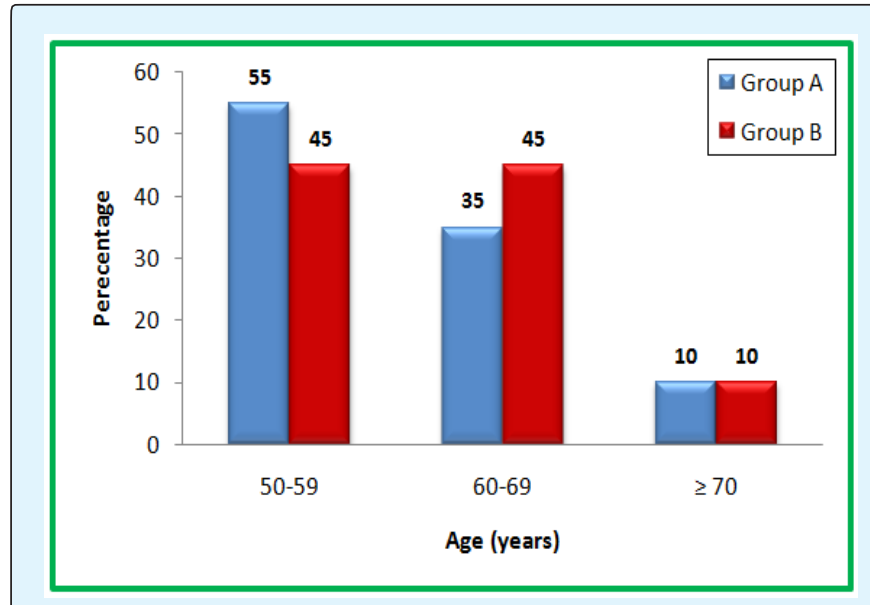

Table 1: Age distribution of study patients among two groups.

Group A (Laparoscopic hand sewn anastomosis) comprised of 20 patients between the ages of 50 and > 70 years (mean age: $59.6 \pm 6.29$ years) maximum number of patients were in the age group of 50-59 years comprising $55 \%$ of group A.

Group B (Laparoscopic stapled anastomosis ) comprised of 20 patients in age range of 50 to 70 years (mean age : 60.9 6.77 years) .Most of the patients were in age range of 50-59 and $60-69$ years of age comprising 45 $\%$ each of group B. P-value was 0.549 .

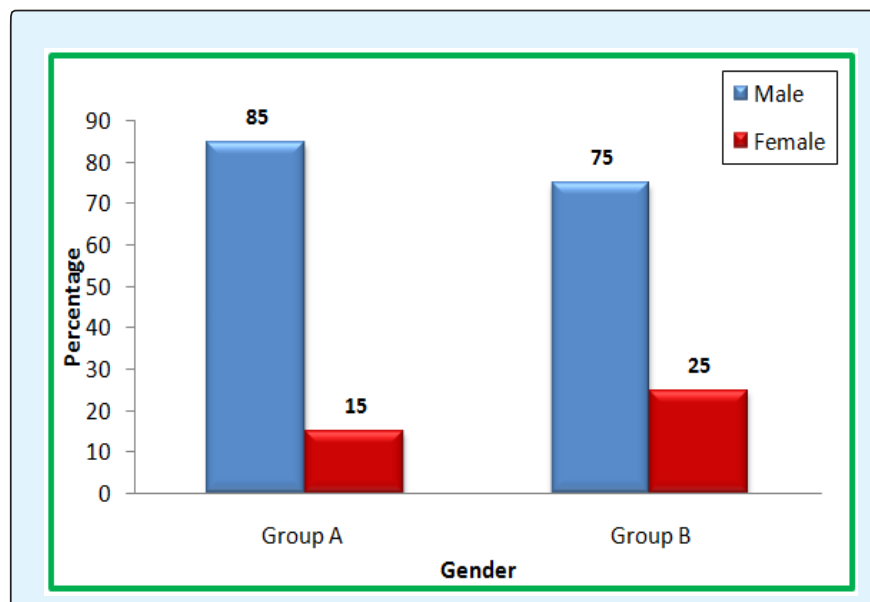

Table 2: Gender distribution of study patients among two groups. 
Group A (Laparoscopic hand sewn anastomosis): 17 males were in group A,comprising of $85 \%$ and 3 females comprising of $15 \%$ of group A.

Group B (Laparoscopic stapled anastomosis): 15 males were in group B, comprising $75 \%$ and 5 females comprising $25 \%$ of group B.

$\mathrm{P}$-value is 0.695

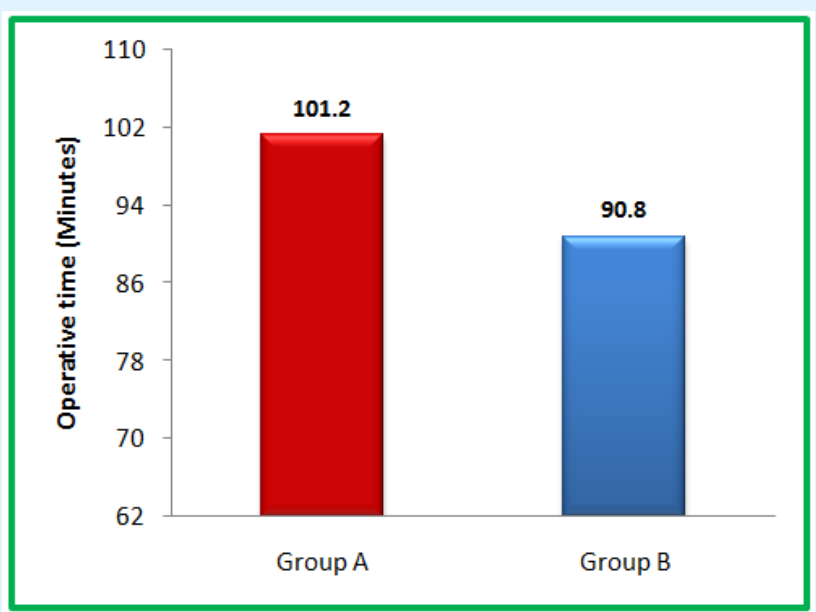

Table 3: Showing operative time (minutes) among two groups.

Group A (Laparoscopic hand sewn anastomosis): The mean operative time in group A is 101.2+/- 8.94. For majority of the patients in group A total operative time ranges from 95-100 (11 patients).

Group B (Laparoscopic stapled anastomosis): The mean operative time in group B was $90.8+/-7.97$.For majority of the patients in group B total operative time ranges from 80-95 (11 patients).

p- Value was $<0.001$.

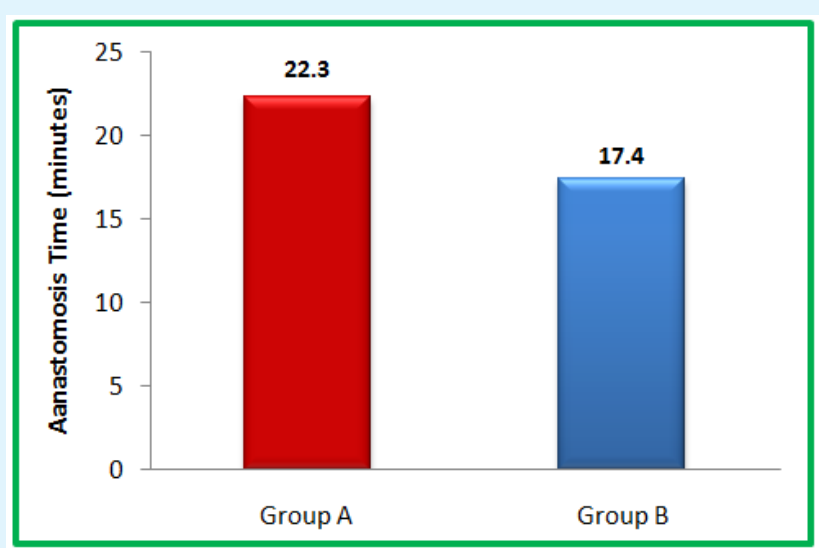

Table 4: Showing anastomosis time (minutes) among two groups.
Group A (Laparoscopic hand sewn anastomosis): The mean time taken for anastomosis in group A was 22.3+/1.86. The anastomosis time ranges from $<20$ to $>25$.

Group B (Laparoscopic stapled anastomosis): The mean time taken for anastomosis in group B was 17.4+/2.48. With 16 patients (80\%) having anastomosis time $<20$ minutes.

p- Value $<0.001$

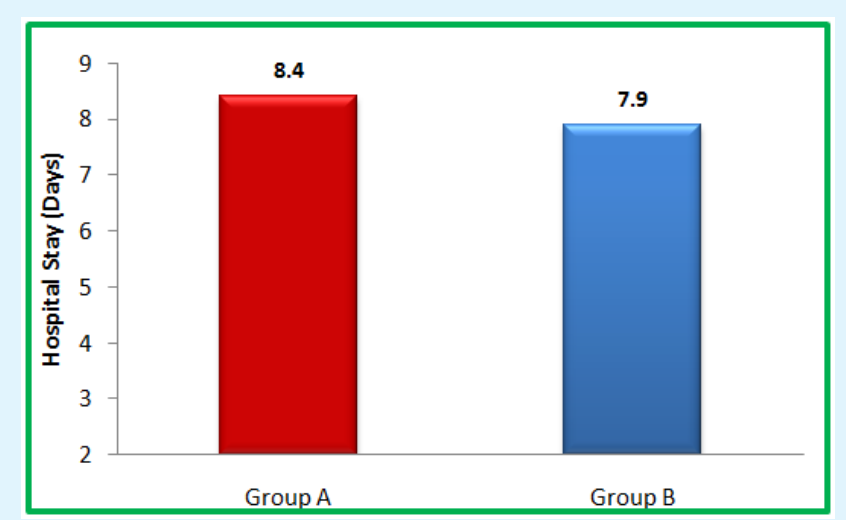

Table 5: Hospital stay (days) among two groups.

Group A (Laparoscopic hand sewn anastomosis): The mean hospital stay in days of group A was 8.4+/-0.89 with majority of the patients having hospital stay of 8 days(40\%). The hospital stay ranged from 7 days to 10 days.

Group B (Laparoscopic stapled anastomosis): The mean hospital stay in days of group B was $7.9+/-0.91$ with majority of the patients having hospital stay of 7 days $(40 \%)$.

The p-value was 0.067 .

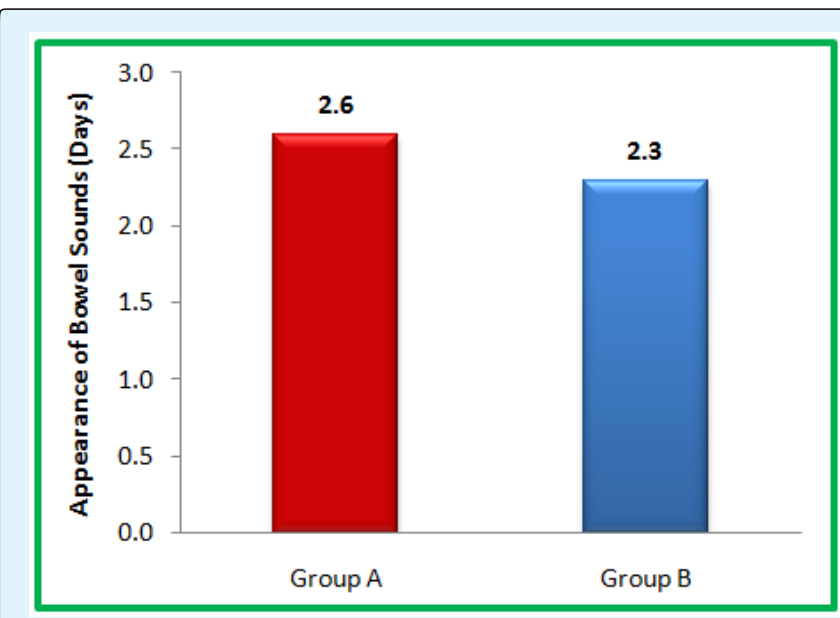

Table 6: Appearance of bowel sounds in study patients among two groups. 
Group A (Laparoscopic hand sewn anastomosis): The Mean time of appearance of bowel sound in group A was $2.6+/-0.59$ with maximum falling in the category of 3 days.

Group B (Laparoscopic stapled anastomosis): The mean time of appearance of bowel sound in group B was $2.3+/-0.47$ with maximum falling in the range of 2 days. P-value 0.076

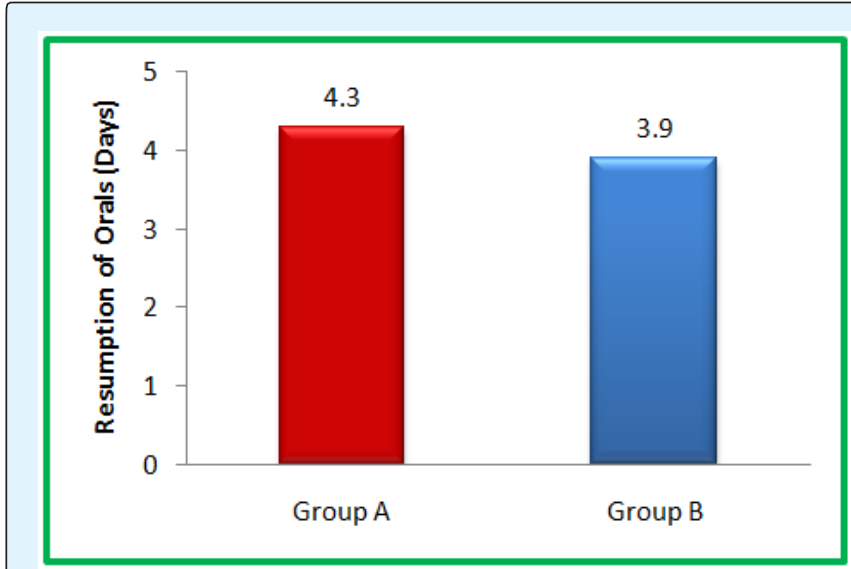

Table 7: Showing resumption of orals among two groups.

Group A (Laparoscopic hand sewn anastomosis): Oral feeding was started in group A on days ranging from 3 to 5.The mean in group A was $4.3+/-0.71$.

Group B (Laparoscopic stapled anastomosis): Oral feeding was started in group B with mean 3.9 +/- 0.72 .

P-value 0.089 .

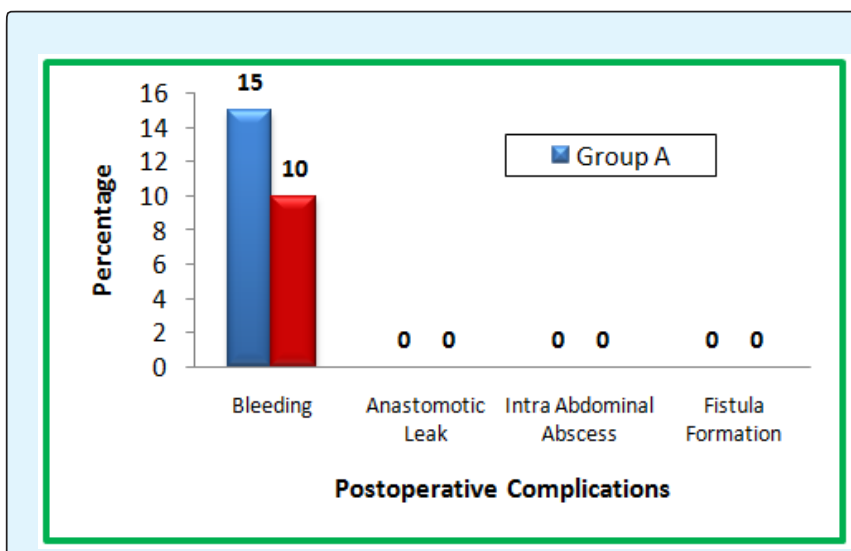

Table 8: Postoperative complications among two groups.
In group A (Laparoscopic hand sewn anastomosis) the complication rate was 15 percent with the only complication being suture line bleed.

In group B (Laparoscopic stapled anastomosis) the only complication was staple line hemorrhage which comprised complication rate of 10 percent.

$\mathrm{P}$-value was 0.633 .
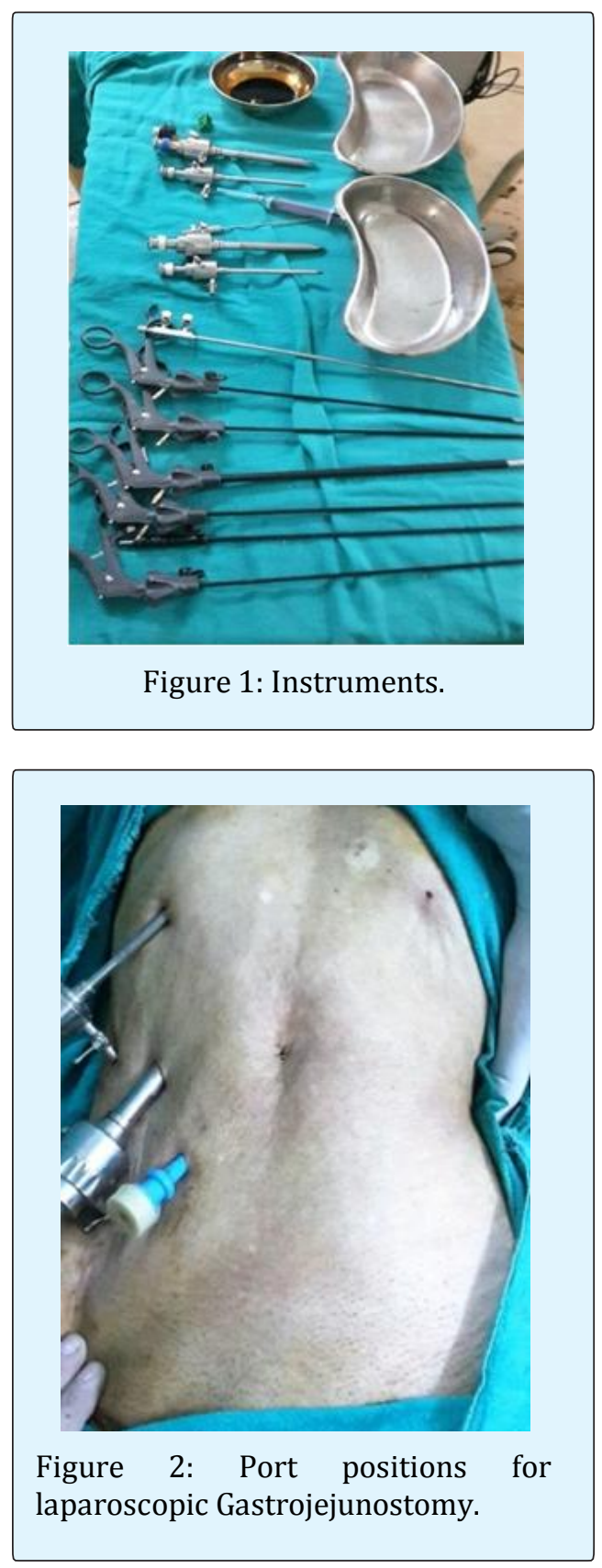

Mushtaq C, et al. A Short Term Outcome of Laparoscopic Hand Sewn and Stapled Gastrojejunostomy in Gastric Outlet Obstruction - An Observational Study. Int J Surg Surgical Tech 2018, 2(2): 000118. 


\section{International Journal of Surgery \& Surgical Techniques}
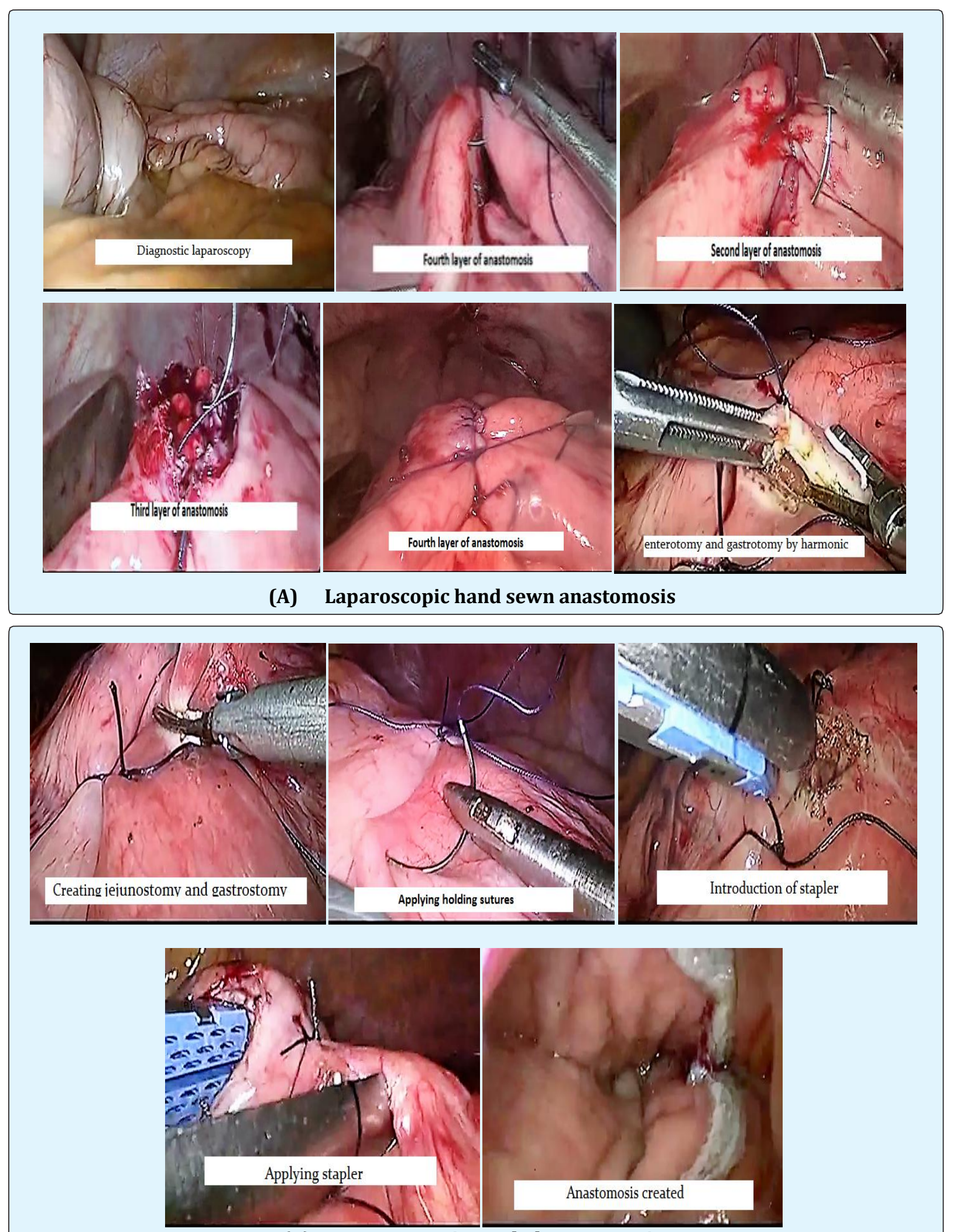

(B) Laparoscopic stapled anastomosis 


\section{International Journal of Surgery \& Surgical Techniques}

\section{Discussion}

With the advent of laparoscopic and minimal access techniques different modalities are in use for the treatment of gastric outlet obstruction from advanced malignancies and benign disorders. Open gastrojejunostomy used to be the only method to relieve gastric outlet obstruction. Owing to the introduction of endoscopic stenting and laparoscopic surgery, lesser and lesser invasive approaches are now more commonly being used. Diagnostic laparoscopy is gaining momentum and acceptance as preliminary modality to be followed in all cases of gastrointestinal malignancy especially carcinoma stomach. It provides a gamut of information about the extent of tumor, biopsy of tumor, MNT staging of the tumor and analysis of malignant ascites if present. In our study, our aims and objectives were defined and conclusive. We routinely followed laparoscopy as a modality for diagnosis and even treatment for unresectable distal gastric tumors. The patients having gastric outlet obstruction with stage 4 disease were taken up for laparoscopic gastrojejunostomy. It was decided to use two techniques of laparoscopic gastrojejunostomy. In one of the techniques we used barbed sutures; copolymer of glycolic acid and trimethylene carbonate ( $\mathrm{v}$ loc, stratafix), vicryl for creating laparoscopic hand sewn anastomosis. In other technique, we used laparoscopic linear stapler with $60 \mathrm{~mm}$ mounted cartridge for creating $6 \mathrm{~cm}$ anastomosis and results were observed keeping in aims and objectives of our study in consideration. While surveying the literature, we found a very few work is published in this concern. In this study, we reviewed our institution's experience of laparoscopic gastrojejunostomy as a treatment modality for GOO.

The results were analyzed and compared with published literature both in India and abroad.

Various parameters were observed. In our study Group A (Laparoscopic hand sewn anastomosis) comprised of 20 patients between the ages of 50 and 70 years (mean age: $59.6 \pm 6.29$ years) while Group B (Laparoscopic stapled anastomosis) comprised of 20 patients in age range of 50 to 70 years (mean age: 60.9 \pm 6.77 years).Most of the patients were in age range of 50-59 years of age in both groups. Study done by Vivek, et al. [12] showed the majority of the study population was between the age group of 40 and 80 years with a maximum number of individuals falling in the group of 51-60 years In study by Linda P. Zhang, et al. [13] in a study a total of 28 patients were reviewed. Average age was 68 (range, 25 to 99). In study by Su Hyun Seo, et al. [14] the mean age of patients in stapled group was $60.1+/-11.7$, the mean age of patients in stapled group was $60.4+/-12$.

In our study the majority of the study population was males in both groups. The study sample showed 17 males and 3 females in Group A (Laparoscopic hand sewn anastomosis) and 15 males and 5 females in Group B (Laparoscopic stapled anastomosis) The P-value was 0.695. In a study done by Hyasinta Jaka, et al. [15] the male female ratio was 3.2: 1 for the malignant gastric outlet obstruction. This difference was statistically significant $(\mathrm{P}<0.001)$.

In study done by Vivek, et al. Gender distribution of the study sample. The majority of the study population was males with a male to female ratio of 3.2:1. The finding was concurrent with studies which suggested males are more commonly affected with GOO and the outnumber females [16].

Laparoscopic Gastrojejunostomy is technically more difficult than open Gastrojejunostomy in creating bypass. Laparoscopy is a less invasive procedure than open surgery. In this study total operative time was taken in minutes. The time was taken from introduction of ports to removal of all ports. In Gastrojejunostomy group, the mean operating time was shortened in the stapler group and the difference was statistically significant ( $p$ value $<0.001$ ).

In study done by Choi YB, et al. [17] on Laparoscopic Gastrojejunostomy for palliation of gastric outlet obstruction in unrespectable gastric cancer the mean operative time for stapled laparoscopic Gastrojejunostomy was $100.5+/-9.8$. In a study done by Higa, et al. [18] the total operative time for creating laparoscopic Gastrojejunostomy hand sewn anastomosis was 60-90 minutes.+/- 9.8 min. In a study done by Linda P. Zhang, et al. the average operative time for laparoscopic stapled Gastrojejunostomy was 170 minutes. Prior studies have shown that operative time decreases as the individual surgeons become more experienced [19]. The operative time is also likely surgeon and patient dependent.

In our study the mean time taken for creating anastomosis was $22.3+/-1.86$ minutes in hand sewn anastomosis and $17.4+/-2.48$ minutes and $p$ - value was $<0.001$ which was significant difference for creating anastomosis. In a study done by Su Hyun Seo, et al. time taken for creating anastomosis by hand sewn method was $15.2+/-2.2$ and stapled anastomosis was $5.7 \pm 0.7$ the $p$ 


\section{International Journal of Surgery \& Surgical Techniques}

value between hand sewn and stapled anastomosis was $<0.001$ which was significant difference.

The hospital length of stay can be as low as 3 days and as high as 14 days $[20,21]$ in laparoscopic Gastrojejunostomy. In our study the mean postoperative hospital stay for hand sewn anastomosis was $8.4+/-0.89$ and in stapled group was $7.9+/-0.91$ and p-value was 0.067 which was insignificant. This total postoperative hospital stay is shorter than the reported length of stay for open cases. Jeurnink, et al. [22] performed a systemic review of published series on GJ between 1996 and 2006. Of the 297 cases of GJ (Gastrojejunostomy), 226 patients had open GJ, and their average length of stay ranged from 8.5 days to 24 days.

In a study done by Su Hyun Seo, et al. the mean postoperative hospital stay in hands sewn $8.0+/-3.8$ and stapled anastomosis was $7.4+/-1.9$ and P- Value was 0.497 this study showed no significant difference between hospital stay in stapled and hand sewn anastomosis.

In a study done by Linda $\mathrm{P}$, et al. the median length of postoperative hospital stay found in this study was 8 days. In our study the mean time of appearance of bowel sounds in hand sewn group was $2.6+/-0.59$ and stapled group was $2.3+/-0.47$ and P-value was 0.076 which was insignificant.

In a study by Su Hyun Seo, et al. appearance of bowel sound was found to be $2.8+/-0.9$ in hand sewn anastomosis and $2.4+/-0.9$ in stapled anastomosis group and $\mathrm{p}$ - value was 0.104 and was insignificant. In a study by Linda $\mathrm{P}$ et al. the appearance of bowel sound in stapled anastomosis was found to be 3 days.

Resumption of orals in hand sewn anastomosis was 4.3 +/- 0.71 and in hand sewn anastomosis was $3.9+/-0.72$ and p- value was 0.089 which was insignificant difference between two groups (Table 7). In a study done by Linda et al the mean days for resumption or orals in laparoscopic Gastrojejunostomy (stapled anastomosis) was found to be 3 days. In a study by Alam, et al. [23] and Kazanjian, et al. [24] both reviewed patients with GOO secondary to inoperable cancer and found the median time to solid food after laparoscopic Gastrojejunostomy to be 4 days. In contrast, open Gastrojejunostomy generally required 7 days before a patient could resume solid food. As we understand the complications are part of any surgery performed. Surprisingly in our study we observed complication rate on a lesser ebb than mentioned in the literature. Among the various complications of laparoscopic Gastrojejunostomy, we found intra operative anastomosis site hemorrhage to the tune of $15 \%$; total of 3 cases, $10 \%$ in laparoscopic stapled anastomosis; total of 2 patients. P-value between two groups was 0.633 which was insignificant difference between two groups. In a study by Rodrigo Gonzalez, et al. [25] in their study of 108 patients with eighty seven patients had hand sewn anastomosis and 21 had stapled anastomosis there was no significant difference in anastomosis related hemorrhage with $\mathrm{p}$ value 0.34 (hand sewn $=2$, stapled $=1$ ). The anastomosis leak is one of the complications of Gastrojejunostomy hand sewn or stapled. It was observed that our study group of patients did not have any anastomosis leakage in contrast to the literature mentioned leak rate of $3.57 \%$ by Linda P. Zhang, et al. in his study, $1.0 \%$ in laparoscopic stapled anastomosis and $1.1 \%$ in laparoscopic hand sewn anastomosis by Frank P. Bendewald [26]. This we possibly attribute to the surgeon expertise and optimum preoperative preparation.

Intra-abdominal abscess and fistula formation is also complication of laparoscopic Gastrojejunostomy mentioned in the literature. However, in our study we did not find any such case with intra-abdominal abscess or fistula formation in our follow-up. It is important to mention that we observed contrast results as regards with complications of the procedure mentioned in the literature. This is possibly because the single surgeon performed all the procedures both laparoscopic hand sewn and stapled anastomosis. The patients were properly optimized with a special reference to the clinical built, hemoglobin level, plasma proteins and preparation of stomach and small bowel.

\section{Summary}

This Prospective study was observational and was aimed to observe the laparoscopic Gastrojejunostomy hand sewn and stapled anastomosis on the basis of various parameters.

- There was no significant difference between the two groups in terms of age.

- Majority of the patients in our study were males in both groups.

- Total operative time was significantly less in laparoscopic Gastrojejunostomy (stapled anastomosis).

- In our study time taken for anastomosis was less in stapled Gastrojejunostomy. 


\section{International Journal of Surgery \& Surgical Techniques}

- There was no significant difference in hospital stay, appearance of bowel sounds, and resumption of orals on both groups.

- The main Intraoperative / postoperative complication were suture line hemorrhage.

\section{Conclusion}

- Stapling devices in surgery are a versatile tool in the armamentarium of a surgeon.

- Anastomosis in stapled gastrojejunostomy takes less time and thus decreases total operative time.

- Laparoscopic hand sewn(suture)anastomosis avoids the use of staplers and suits to the patients from developing countries

\section{References}

1. Samad A, Whanzada TW, Shoukat I (2007) Gastric outlet obstruction: Change in etiology. Pak J Surg 23(1): 29-32.

2. Johnson CD (1995) Gastric outlet obstruction malignant until proved otherwise. Am J Gastroenterol 90(10): 1740.

3. Tendler DA (2002) malignant gastric outlet obstruction: Bridging another divide. Am J Gastroenterol 97(1): 4-6.

4. Lans HS, Stein IF, Meyer KA (1952) Electrolyte abnormalities in pyloric obstruction resulting from peptic ulcer or gastric carcinoma. Ann Surg 135(4): 441-453.

5. Shone DN, Nikoomanesh P, Smith-Meek MM, Bender JS (1995) Malignancy is the most common cause of gastric outlet obstruction in the era of $\mathrm{H} 2$ blockers. Am J Gastroenterol 90(10): 1769-1770.

6. Adler DG, Baron TH (2002) Endoscopic palliation of malignant gastric outlet obstruction using selfexpanding metal stents: experience in 36 patients. Am J Gastroenterol 97(1): 72-78.

7. Pasini F, Fraccon AP, DE Manzoni G (2011) The role of chemotherapy in metastatic gastric cancer. Anticancer Res 31(10): 3543-3554.

8. Japanese Gastric Cancer Association (2011) Japanese gastric cancer treatment guidelines 2010 (ver. 3) Gastric Cancer 14: 113-123.
9. Abdel-Salam WN, Katri KM, Bessa SS, El-Kayal el-SA (2009) Laparoscopic-assisted truncalvagotomy and gastro-jejunostomy: trial of simplification. J Laparoendosc Adv Surg Tech A 19(2): 125-127.

10. Siu WT, Tang CN, Law BK, Chau CH, Yau KK, et al. (2004) Vagotomy and gastrojejunostomy for benign gastric outlet obstruction. J Laparoendosc Adv Surg Tech A. 2004 Oct. 14(5): 266-269.

11. LagmanRL, Davis MP, LeGrand SB, Walsh D (2005) Common symptoms in advanced cancer. SurgClin North Am 85(2): 237-255.

12. Sukumar V, Ravindran C, Prasad RV (2015) Demographic and etiological patterns of gastric outlet obstruction in Kerala, South India. N Am J Med Sci 7(9): 403-406.

13. Linda P Zhang, Parissa Tabrizian, Scott Nguyen, Dana Telem, Celia Divino (2011) Laparoscopic Gastrojejunostomy for the Treatment of Gastric Outlet Obstruction. JSLS 15(2): 169-173.

14. Su Hyun Seo, Ki Han Kim, Min Chan Kim, Hong Jo Choi, GhapJoong Jung (2012) Comparative Study of Hand-Sutured versus Circular Stapled Department of Surgery, Dong-A University College of Medicine, Busan, Korea J Gastric Cancer 12(2): 120-125.

15. Hyasinta Jaka, Mabula D Mchembe, Peter F Rambau, PhillipoL Chalya (2013) Gastric outlet obstruction at Bugando Medical Centre in Northwestern Tanzania: a prospective review of 184 cases. BMC Surgery 13: 41.

16. Jaka H, Mchembe MD, Rambau PF, Chalya PL (2013) Gastric outlet obstruction at Bugando Medical Centre in Northwestern Tanzania: a prospective review of 184 cases. BMC Surg 13: 41.

17. Choi YB (2002) Laparoscopic gatrojejunostomy for palliation of gastric outlet obstruction in unresectable gastric cancer. Surgendosc 16(11): 1620-1626.

18. Higa KD, Boone KB, Ho T, Davies OG (2000) laparoscopic roux-en-y gastric bypass for morbid obesity: technique and preliminary results of our first 400 patients. Arc surg 135(9): 1029-1034.

19. Pournaras DJ, Jafferbhoy S, Titcomb DR, Humadi S, Edmond JR, et al. (2010) Three hundred laparoscopic Roux-en-Y gastric bypasses: managing the learning 
curve in higher risk patients. Obes Surg 20(3): 290294.

20. Navarra G, Musolino C, Venneri A, De Marco ML, Bartolotta M (2006) Palliative antecoliciso peristaltic gastrojejunostomy: a randomized controlled trial comparing open and laparoscopic approaches. Surg Endosc 20(12): 1831-1834.

21. Al-Rashedy M, Dadibhai M, Shareif A, Khandelwal MI, Ballester P, et al. (2005) Laparoscopic gastric bypass for gastric outlet obstruction is associated with smoother, faster recovery and shorter hospital stay compared with open surgery. J Hepatobiliary Pancreat Surg 12(6): 474-478.

22. Jeurnink SM, van Eijck CH, Steyerberg EW, Kuipers EJ, Siersema PD (2007) Stent versus gastrojejunostomy for the palliation of gastric outlet obstruction: a systematic review. BMC Gastroenterol 8: 18.
23. Alam TA, Baines M, Parker MC (2003) the management of gastric outlet obstruction secondary to inoperable cancer. Surg Endosc 17(2): 320-323.

24. Kazanjian KK, Reber HA, Hines OJ (2004) Laparoscopic Gastrojejunostomy for gastric outlet obstruction in pancreatic cancer. Am Surg 70(10): 910-913.

25. Gonzalez R, Lin E, Venkatesh KR, Bowers SP, Smith CD (2003) Gastrojejunostomy During Laparoscopic Gastric Bypass - Analysis of 3 Techniques. Arch Surg 138(2): 181-184.

26. Bendewald FP, Choi JN, Blythe LS, Selzer DJ, Ditslear $\mathrm{JH}$, et al. (2011) Comparison of hand-sewn, linearstapled, and circular-stapled gastrojejunostomy in laparoscopic Roux-en-Y gastric bypass. Obes Surg 21(11): 1671-1675. 